\title{
The Regularity of Functions on Dual Split Quaternions in Clifford Analysis
}

\author{
Ji Eun Kim and Kwang Ho Shon \\ Department of Mathematics, Pusan National University, Busan 609-735, Republic of Korea \\ Correspondence should be addressed to Kwang Ho Shon; khshon@pusan.ac.kr
}

Received 28 January 2014; Accepted 2 April 2014; Published 17 April 2014

Academic Editor: Zong-Xuan Chen

Copyright ( 92014 J. E. Kim and K. H. Shon. This is an open access article distributed under the Creative Commons Attribution License, which permits unrestricted use, distribution, and reproduction in any medium, provided the original work is properly cited.

This paper shows some properties of dual split quaternion numbers and expressions of power series in dual split quaternions and provides differential operators in dual split quaternions and a dual split regular function on $\Omega \subset \mathbb{C}^{2} \times \mathbb{C}^{2}$ that has a dual split Cauchy-Riemann system in dual split quaternions.

\section{Introduction}

Hamilton introduced quaternions, extending complex numbers to higher spatial dimensions in differential geometry (see [1]). A set of quaternions can be represented as

$$
\mathscr{H}=\left\{z=x_{0}+x_{1} i+x_{2} j+x_{3} k: x_{m} \in \mathbb{R}, m=0,1,2,3\right\},
$$

where $i^{2}=j^{2}=k^{2}=-1, i j k=-1$, and $\mathbb{R}$ denotes the set of real numbers. Cockle [2] introduced a set of split quaternions as

$$
\mathcal{S}=\left\{z=x_{0}+x_{1} e_{1}+x_{2} e_{2}+x_{3} e_{3}: x_{m} \in \mathbb{R}, m=0,1,2,3\right\},
$$

where $e_{1}^{2}=-1, e_{2}^{2}=e_{3}^{2}=1$, and $e_{1} e_{2} e_{3}=1$. A set of split quaternions is noncommutative and contains zero divisors, nilpotent elements, and nontrivial idempotents (see $[3,4])$. Previous studies have examined the geometric and physical applications of split quaternions, which are required in solving split quaternionic equations (see $[5,6]$ ). Inoguchi [7] reformulated the Gauss-Codazzi equations in forms consistent with the theory of integrable systems in the Minkowski 3-space for split quaternion numbers.

A dual quaternion can be represented in a form reflecting an ordinary quaternion and a dual symbol. Because dual-quaternion algebra is constructed from real eightdimensional vector spaces and an ordered pair of quaternions, dual quaternions are used in computer vision applications. Kenwright [8] provided the characteristics of dual quaternions, and Pennestrì and Stefanelli [9] examined some properties by using dual quaternions. Son $[10,11]$ offered an extension problem for solutions of partial differential equations and generalized solutions for the Riesz system. By using properties of Hamilton operators, Kula and Yayli [4] defined dual split quaternions and gave some properties of the screw motion in the Minkowski 3-space, showing that $\mathscr{H}$ has a rotation with unit split quaternions in $\mathscr{H}$ and a scalar product that allows it to be identified with the semi-Euclidean space for split quaternion numbers.

It was shown (see $[12,13])$ that any complex-valued harmonic function $f_{1}$ in a pseudoconvex domain $D$ of $\mathbb{C}^{2} \times \mathbb{C}^{2}$, $\mathbb{C}$ being the set of complex numbers, has a conjugate function $f_{2}$ in $D$ such that the quaternion-valued function $f_{1}+f_{2} j$ is hyperholomorphic in $D$ and gave a regeneration theorem in a quaternion analysis in view of complex and Clifford analysis. In addition, we $[14,15]$ provided a new expression of the quaternionic basis and a regular function on reduced quaternions by associating hypercomplex numbers $e_{1}$ and $e_{2}$. We [16] investigated the existence of hyperconjugate harmonic functions of an octonion number system, and we $[17,18]$ obtained some regular functions with values in dual quaternions and researched an extension problem for properties 
of regular functions with values in dual quaternions and some applications for such problems.

This paper provides a regular function and some properties of differential operators in dual split quaternions. In addition, we research some equivalent conditions for CauchyRiemann systems and expressions of power series in dual split quaternions from the definition of dual split regular on an open set $\Omega \subset \mathbb{C}^{2} \times \mathbb{C}^{2}$.

\section{Preliminaries}

A dual number $A$ has the form $a+\varepsilon b$, where $a$ and $b$ are real numbers and $\varepsilon$ is a dual symbol subject to the rules

$$
\varepsilon \neq 0, \quad 0 \varepsilon=\varepsilon 0=0, \quad 1 \varepsilon=\varepsilon 1=\varepsilon, \quad \varepsilon^{2}=0,
$$

and a split quaternion $q \in \mathcal{S}$ is an expression of the form

$$
q=x_{0}+x_{1} e_{1}+x_{2} e_{2}+x_{3} e_{3}
$$

where $x_{m} \in \mathbb{R}(m=0,1,2,3)$ and $e_{r}(r=1,2,3)$ are split quaternionic units satisfying noncommutative multiplication rules (for split quaternions, see [1]):

$$
\begin{aligned}
& e_{1}^{2}=-1, \quad e_{2}^{2}=e_{3}^{2}=1, \\
& e_{1} e_{2}=-e_{2} e_{1}=e_{3}, \quad e_{2} e_{3}=-e_{3} e_{2}=-e_{1}, \\
& e_{3} e_{1}=-e_{1} e_{3}=e_{2} .
\end{aligned}
$$

Similarly, a dual split quaternion $z$ can be written as

$$
\mathscr{D}(\mathcal{S})=\left\{z \mid z=p_{0}+\varepsilon p_{1}, p_{r} \in \mathcal{S}, r=0,1\right\},
$$

which has elements of the following form:

$$
\begin{aligned}
z= & \left\{\left(x_{0}+x_{1} e_{1}\right)+\left(x_{2}+x_{3} e_{1}\right) e_{2}\right\} \\
& +\varepsilon\left\{\left(y_{0}+y_{1} e_{1}\right)+\left(y_{2}+y_{3} e_{1}\right) e_{2}\right\} \\
= & \left(z_{0}+z_{1} e_{2}\right)+\varepsilon\left(z_{2}+z_{3} e_{2}\right) \\
= & p_{0}+\varepsilon p_{1},
\end{aligned}
$$

where $p_{0}=z_{0}+z_{1} e_{2}$ and $p_{1}=z_{2}+z_{3} e_{2}$ are split quaternion components, $z_{0}=x_{0}+x_{1} e_{1}, z_{1}=x_{2}+x_{3} e_{1}, z_{2}=y_{0}+y_{1} e_{1}$, and $z_{3}=y_{2}+y_{3} e_{1}$ are usual complex numbers, and $x_{m}, y_{m} \in$ $\mathbb{R}(m=0,1,2,3)$. The multiplication of split quaternionic units with a dual symbol is commutative $\varepsilon e_{r}=e_{r} \varepsilon(r=$ $1,2,3)$. However, by properties of split quaternionic unit,

$$
\begin{gathered}
z_{k} e_{r}=e_{r} z_{k} \quad(k=0,1,2,3, r=0,1), \\
z_{k} e_{r}=e_{r} \overline{z_{k}} \quad(k=0,1,2,3, r=2,3), \\
e_{r} p_{k} \neq p_{k} e_{r}, \quad e_{r} p_{k}=p_{(k r)} e_{r} \quad(r=1,2,3, k=0,1),
\end{gathered}
$$

where

$$
\begin{aligned}
& p_{(01)}=z_{0}-z_{1} e_{2}=x_{0}+x_{1} e_{1}-x_{2} e_{2}-x_{3} e_{3}, \\
& p_{(02)}=\overline{z_{0}}+\overline{z_{1}} e_{2}=x_{0}-x_{1} e_{1}+x_{2} e_{2}-x_{3} e_{3}, \\
& p_{(03)}=\overline{z_{0}}-\overline{z_{1}} e_{2}=x_{0}-x_{1} e_{1}-x_{2} e_{2}+x_{3} e_{3}, \\
& p_{(11)}=z_{2}-z_{3} e_{2}=y_{0}+y_{1} e_{1}-y_{2} e_{2}-y_{3} e_{3}, \\
& p_{(12)}=\overline{z_{2}}+\overline{z_{3}} e_{2}=y_{0}-y_{1} e_{1}+y_{2} e_{2}-y_{3} e_{3}, \\
& p_{(13)}=\overline{z_{2}}-\overline{z_{3}} e_{2}=y_{0}-y_{1} e_{1}-y_{2} e_{2}+y_{3} e_{3},
\end{aligned}
$$

with $\overline{z_{0}}=x_{0}-x_{1} e_{1}, \overline{z_{1}}=x_{2}-x_{3} e_{1}, \overline{z_{2}}=y_{0}-y_{1} e_{1}$, and $\overline{z_{3}}=y_{2}-y_{3} e_{1}$. For instance,

$$
\begin{aligned}
e_{2} p_{0} & =e_{2}\left(x_{0}+x_{1} e_{1}+x_{2} e_{2}+x_{3} e_{3}\right) \\
& =\left(x_{0}-x_{1} e_{1}+x_{2} e_{2}-x_{3} e_{3}\right) e_{2}=p_{(02)} e_{2}, \\
e_{1} p_{1} & =e_{1}\left(y_{0}+y_{1} e_{1}+y_{2} e_{2}+y_{3} e_{3}\right) \\
& =\left(y_{0}+y_{1} e_{1}-y_{2} e_{2}-y_{3} e_{3}\right) e_{1}=p_{(11)} e_{1} .
\end{aligned}
$$

Because of the properties of the eight-unit equality, the addition and subtraction of dual split quaternions are governed by the rules of ordinary algebra. Here the symbol $p_{(k r)}$ is used by just enumerating $r$ and $k$, not $r$ times $k$. For example, $p_{(22)} \neq p_{4}$ and $p_{22}=p_{4}$

For any two elements $z=p_{0}+\varepsilon p_{1}$ and $w=q_{0}+\varepsilon q_{1}$ of $\mathscr{D}(\mathcal{S})$, where $q_{0}=\sum_{r=0}^{3} s_{r} e_{r}$ and $q_{1}=\sum_{r=0}^{3} t_{r} e_{r}$ are split quaternion components and $s_{r}, t_{r} \in \mathbb{R}(r=0,1,2,3)$, their noncommutative product is given by

$$
z w=\left(p_{0}+\varepsilon p_{1}\right)\left(q_{0}+\varepsilon q_{1}\right)=p_{0} q_{0}+\varepsilon\left(p_{0} q_{1}+p_{1} q_{0}\right) .
$$

The conjugation $z^{*}$ of $z$ and the corresponding modulus $z z^{*}$ in $\mathscr{D}(\mathcal{S})$ are defined by

$$
\begin{aligned}
z^{*} & =p_{0}^{*}+\varepsilon p_{1}^{*}, \\
z z^{*} & =z^{*} z=p_{0} p_{0}^{*}+\varepsilon\left(p_{0} p_{1}^{*}+p_{1} p_{0}^{*}\right) \\
& =\left(z_{0} \overline{z_{0}}-z_{1} \overline{z_{1}}\right)+2 \varepsilon\left(z_{0} \overline{z_{2}}-z_{1} \overline{z_{3}}\right) \\
& =\sum_{r=0}^{1}\left\{\left(x_{r}^{2}-x_{r+2}^{2}\right)+\varepsilon\left(x_{r} y_{r}-x_{r+2} y_{r+2}\right)\right\},
\end{aligned}
$$

where $p_{0}^{*}=\overline{z_{0}}-z_{1} e_{2}$ and $p_{1}^{*}=\overline{z_{2}}-z_{3} e_{2}$.

Lemma 1. For all $z \in \mathscr{D}(\mathcal{S})$ and $n \in \mathbb{N}:=\{1,2,3, \ldots\}$, we have

$$
z^{n}=p_{0}^{n}+\varepsilon \sum_{k=1}^{n} p_{0}^{n-k} p_{1} p_{0}^{k-1}
$$


Proof. If $n=1$, then (13) is trivial. Now suppose that this holds for some $n \in \mathbb{N}$. Then, as desired,

$$
\begin{aligned}
z^{n+1} & =z z^{n}=z\left(p_{0}^{n}+\varepsilon \sum_{k=1}^{n} p_{0}^{n-k} p_{1} p_{0}^{k-1}\right) \\
& =p_{0}^{n+1}+\varepsilon \sum_{k=1}^{n} p_{0}^{n-k+1} p_{1} p_{0}^{k-1}+\varepsilon p_{1} p_{0}^{n} \\
& =p_{0}^{n+1}+\varepsilon \sum_{k=1}^{n+1} p_{0}^{n+1-k} p_{1} p_{0}^{k-1} .
\end{aligned}
$$

By the principle of mathematical induction, (13) holds for all $n \in \mathbb{N}$.

Let $\Omega$ be an open subset of $\mathbb{C}^{2} \times \mathbb{C}^{2}$. Then the function $f: \Omega \rightarrow \mathscr{D}(\mathcal{S})$ can be expressed as

$$
f(z)=f\left(p_{0}, p_{1}\right)=f_{0}\left(p_{0}, p_{1}\right)+\varepsilon f_{1}\left(p_{0}, p_{1}\right),
$$

where the component functions $f_{r}: \Omega \rightarrow \mathcal{S}(r=0,1)$ are split quaternionic-valued functions. The component functions $f_{r}(r=0,1)$ are

$$
\begin{aligned}
f_{0}\left(p_{0}, p_{1}\right) & =f_{0}\left(z_{0}, z_{1}, z_{2}, z_{3}\right) \\
& =g_{0}\left(z_{0}, z_{1}, z_{2}, z_{3}\right)+g_{1}\left(z_{0}, z_{1}, z_{2}, z_{3}\right) e_{2}, \\
f_{1}\left(p_{0}, p_{1}\right) & =f_{1}\left(z_{0}, z_{1}, z_{2}, z_{3}\right) \\
& =g_{2}\left(z_{0}, z_{1}, z_{2}, z_{3}\right)+g_{3}\left(z_{0}, z_{1}, z_{2}, z_{3}\right) e_{2},
\end{aligned}
$$

where $g_{k}=u_{2 k}+u_{2 k+1} e_{1}(k=0,1)$ and $g_{k}=v_{2 k-4}+$ $v_{2 k-3} e_{1}(k=2,3)$ are complex-valued functions, and $u_{r}$ and $v_{r}(r=0,1,2,3)$ are real-valued functions.

Now, we let differential operators $D_{1}$ and $D_{2}$ be defined on $\mathscr{D}(\mathcal{S})$ as

$$
D_{1}:=D_{(11)}+\varepsilon D_{(12)}, \quad D_{2}:=D_{(21)}+\varepsilon D_{(22)} .
$$

Then the conjugate operators $D_{1}^{*}$ and $D_{2}^{*}$ are

$$
D_{1}^{*}=D_{(11)}^{*}+\varepsilon D_{(12)}^{*}, \quad D_{2}^{*}=D_{(21)}^{*}+\varepsilon D_{(22)}^{*},
$$

where

$$
\begin{aligned}
D_{(11)} & =\frac{\partial}{\partial z_{0}}+\frac{\partial}{\partial \bar{z}_{1}} e_{2}=\frac{1}{2}\left(\frac{\partial}{\partial x_{0}}-\frac{\partial}{\partial x_{1}} e_{1}+\frac{\partial}{\partial x_{2}} e_{2}+\frac{\partial}{\partial x_{3}} e_{3}\right), \\
D_{(12)} & =\frac{\partial}{\partial z_{2}}+\frac{\partial}{\partial \bar{z}_{3}} e_{2}=\frac{1}{2}\left(\frac{\partial}{\partial y_{0}}-\frac{\partial}{\partial y_{1}} e_{1}+\frac{\partial}{\partial y_{2}} e_{2}+\frac{\partial}{\partial y_{3}} e_{3}\right), \\
D_{(21)} & =\frac{\partial}{\partial z_{0}}+\frac{1}{2} \frac{\partial}{\partial z_{1}} e_{2} \\
& =\frac{1}{2}\left(\frac{\partial}{\partial x_{0}}-\frac{\partial}{\partial x_{1}} e_{1}+\frac{1}{2} \frac{\partial}{\partial x_{2}} e_{2}-\frac{1}{2} \frac{\partial}{\partial x_{3}} e_{3}\right),
\end{aligned}
$$

$$
\begin{aligned}
D_{(22)} & =\frac{\partial}{\partial z_{2}}+\frac{1}{2} \frac{\partial}{\partial z_{3}} e_{2} \\
& =\frac{1}{2}\left(\frac{\partial}{\partial y_{0}}-\frac{\partial}{\partial y_{1}} e_{1}+\frac{1}{2} \frac{\partial}{\partial y_{2}} e_{2}-\frac{1}{2} \frac{\partial}{\partial y_{3}} e_{3}\right), \\
D_{(11)}^{*} & =\frac{\partial}{\partial \overline{z_{0}}}-\frac{\partial}{\partial \overline{z_{1}}} e_{2}=\frac{1}{2}\left(\frac{\partial}{\partial x_{0}}+\frac{\partial}{\partial x_{1}} e_{1}-\frac{\partial}{\partial x_{2}} e_{2}-\frac{\partial}{\partial x_{3}} e_{3}\right), \\
D_{(12)}^{*} & =\frac{\partial}{\partial \overline{z_{2}}}-\frac{\partial}{\partial \overline{z_{3}}} e_{2}=\frac{1}{2}\left(\frac{\partial}{\partial y_{0}}+\frac{\partial}{\partial y_{1}} e_{1}-\frac{\partial}{\partial y_{2}} e_{2}-\frac{\partial}{\partial y_{3}} e_{3}\right), \\
D_{(21)}^{*} & =\frac{\partial}{\partial \overline{z_{0}}}-\frac{1}{2} \frac{\partial}{\partial z_{1}} e_{2} \\
& =\frac{1}{2}\left(\frac{\partial}{\partial x_{0}}+\frac{\partial}{\partial x_{1}} e_{1}-\frac{1}{2} \frac{\partial}{\partial x_{2}} e_{2}+\frac{1}{2} \frac{\partial}{\partial x_{3}} e_{3}\right), \\
D_{(22)}^{*} & =\frac{\partial}{\partial \overline{z_{2}}}-\frac{1}{2} \frac{\partial}{\partial z_{3}} e_{2} \\
& =\frac{1}{2}\left(\frac{\partial}{\partial y_{0}}+\frac{\partial}{\partial y_{1}} e_{1}-\frac{1}{2} \frac{\partial}{\partial y_{2}} e_{2}+\frac{1}{2} \frac{\partial}{\partial y_{3}} e_{3}\right)
\end{aligned}
$$

act on $\mathscr{D}(\mathcal{S})$. These operators are called corresponding Cauchy-Riemann operators in $\mathscr{D}(\mathcal{S})$, where $\partial / \partial z_{r}$ and $\partial / \partial \overline{z_{r}}(r=0,1,2,3)$ are usual differential operators used in the complex analysis.

Remark 2. From the definition of differential operators on $\mathscr{D}(\mathcal{S})$

$$
\begin{aligned}
D_{r} f & =\left(D_{(r 1)}+\varepsilon D_{(r 2)}\right)\left(f_{0}+\varepsilon f_{1}\right) \\
& =D_{(r 1)} f_{0}+\varepsilon\left(D_{(r 1)} f_{1}+D_{(r 2)} f_{0}\right), \\
D_{r}^{*} f & =\left(D_{(r 1)}^{*}+\varepsilon D_{(r 2)}^{*}\right)\left(f_{0}+\varepsilon f_{1}\right) \\
& =D_{(r 1)}^{*} f_{0}+\varepsilon\left(D_{(r 1)}^{*} f_{1}+D_{(r 2)}^{*} f_{0}\right),
\end{aligned}
$$

where $r=1,2$.

Definition 3. Let $\Omega$ be an open set in $\mathbb{C}^{2} \times \mathbb{C}^{2}$. A function $f=$ $f_{0}+\varepsilon f_{1}$ is called an $L_{r}$ (resp., $\left.R_{r}\right)$-regular function $(r=1,2)$ on $\Omega$ if the following two conditions are satisfied:

(i) $f_{k}(k=0,1)$ are continuously differential functions on $\Omega$, and

(ii) $D_{r}^{*} f(z)=0$ (resp., $\left.f(z) D_{r}^{*}=0\right)$ on $\Omega(r=1,2)$.

In particular, the equation $D_{1}^{*} f(z)=0$ of Definition 3 is equivalent to

$$
D_{(11)}^{*} f_{0}=0, \quad D_{(12)}^{*} f_{0}+D_{(11)}^{*} f_{1}=0 .
$$


In addition,

$$
\begin{aligned}
& \frac{\partial g_{0}}{\partial \overline{z_{0}}}-\frac{\partial \overline{g_{1}}}{\partial \overline{z_{1}}}=0, \quad \frac{\partial g_{1}}{\partial \overline{z_{0}}}-\frac{\partial \overline{g_{0}}}{\partial \overline{z_{1}}}=0, \\
& \frac{\partial g_{2}}{\partial \overline{z_{0}}}+\frac{\partial g_{0}}{\partial \overline{z_{2}}}-\frac{\partial \overline{g_{3}}}{\partial \overline{z_{1}}}-\frac{\partial \overline{g_{1}}}{\partial \overline{z_{3}}}=0 \\
& \frac{\partial g_{3}}{\partial \overline{z_{0}}}+\frac{\partial g_{1}}{\partial \overline{z_{2}}}-\frac{\partial \overline{g_{2}}}{\partial \overline{z_{1}}}-\frac{\partial \overline{g_{0}}}{\partial \overline{z_{3}}}=0
\end{aligned}
$$

Concretely, the following system is obtained:

$$
\begin{gathered}
\frac{\partial u_{0}}{\partial x_{0}}-\frac{\partial u_{1}}{\partial x_{1}}-\frac{\partial u_{2}}{\partial x_{2}}-\frac{\partial u_{3}}{\partial x_{3}}=0 \\
\frac{\partial u_{1}}{\partial x_{0}}+\frac{\partial u_{0}}{\partial x_{1}}-\frac{\partial u_{2}}{\partial x_{3}}+\frac{\partial u_{3}}{\partial x_{2}}=0 \\
\frac{\partial u_{2}}{\partial x_{0}}-\frac{\partial u_{3}}{\partial x_{1}}-\frac{\partial u_{0}}{\partial x_{2}}-\frac{\partial u_{1}}{\partial x_{3}}=0 \\
\frac{\partial u_{3}}{\partial x_{0}}+\frac{\partial u_{2}}{\partial x_{1}}-\frac{\partial u_{0}}{\partial x_{3}}+\frac{\partial u_{1}}{\partial x_{2}}=0 \\
\frac{\partial u_{0}}{\partial y_{0}}-\frac{\partial u_{1}}{\partial y_{1}}-\frac{\partial u_{2}}{\partial y_{2}}-\frac{\partial u_{3}}{\partial y_{3}}+\frac{\partial v_{0}}{\partial x_{0}} \\
+\frac{\partial v_{1}}{\partial x_{1}}-\frac{\partial v_{2}}{\partial x_{2}}-\frac{\partial v_{3}}{\partial x_{3}}=0 \\
\frac{\partial x_{3}}{\partial y_{0}}+\frac{\partial v_{0}}{\partial x_{3}}+\frac{\partial v_{1}}{\partial x_{2}}=0 \\
\frac{\partial u_{1}}{\partial y_{0}}+\frac{\partial u_{0}}{\partial y_{1}}-\frac{\partial u_{2}}{\partial y_{3}}+\frac{\partial u_{3}}{\partial y_{2}}+\frac{\partial v_{1}}{\partial x_{0}} \\
+\frac{\partial v_{0}}{\partial x_{1}}-\frac{\partial v_{2}}{\partial x_{3}}+\frac{\partial v_{3}}{\partial x_{2}}=0 \\
\frac{\partial x_{2}}{\partial x_{2}}-\frac{\partial u_{1}}{\partial y_{3}}+\frac{\partial v_{2}}{\partial x_{0}}
\end{gathered}
$$

The above systems (23) and (24) are corresponding CauchyRiemann systems in $\mathscr{D}(\mathcal{S})$. Similarly, the equation $D_{2}^{*} f(z)=$ 0 of Definition 3 is equivalent to

$$
D_{(21)}^{*} f_{0}=0, \quad D_{(22)}^{*} f_{0}+D_{(21)}^{*} f_{1}=0 \text {. }
$$

Then,

$$
\begin{aligned}
& \frac{\partial g_{0}}{\partial \overline{z_{0}}}-\frac{1}{2} \frac{\partial \overline{g_{1}}}{\partial z_{1}}=0, \quad \frac{\partial g_{1}}{\partial \overline{z_{0}}}-\frac{1}{2} \frac{\partial \overline{g_{0}}}{\partial z_{1}}=0, \\
& \frac{\partial g_{2}}{\partial \overline{z_{0}}}+\frac{\partial g_{0}}{\partial \overline{z_{2}}}-\frac{1}{2} \frac{\partial \overline{g_{3}}}{\partial z_{1}}-\frac{1}{2} \frac{\partial \overline{g_{1}}}{\partial z_{3}}=0, \\
& \frac{\partial g_{3}}{\partial \overline{z_{0}}}+\frac{\partial g_{1}}{\partial \overline{z_{2}}}-\frac{1}{2} \frac{\partial \overline{g_{2}}}{\partial z_{1}}-\frac{1}{2} \frac{\partial \overline{g_{0}}}{\partial z_{3}}=0 .
\end{aligned}
$$

Concretely, the following system is obtained:

$$
\begin{gathered}
\frac{\partial u_{0}}{\partial x_{0}}-\frac{\partial u_{1}}{\partial x_{1}}-\frac{1}{2} \frac{\partial u_{2}}{\partial x_{2}}+\frac{1}{2} \frac{\partial u_{3}}{\partial x_{3}}=0 \\
\frac{\partial u_{1}}{\partial x_{0}}+\frac{\partial u_{0}}{\partial x_{1}}+\frac{1}{2} \frac{\partial u_{2}}{\partial x_{3}}+\frac{1}{2} \frac{\partial u_{3}}{\partial x_{2}}=0 \\
\frac{\partial u_{2}}{\partial x_{0}}-\frac{\partial u_{3}}{\partial x_{1}}-\frac{1}{2} \frac{\partial u_{0}}{\partial x_{2}}+\frac{1}{2} \frac{\partial u_{1}}{\partial x_{3}}=0 \\
\frac{\partial u_{3}}{\partial x_{0}}+\frac{\partial u_{2}}{\partial x_{1}}+\frac{1}{2} \frac{\partial u_{0}}{\partial x_{3}}+\frac{1}{2} \frac{\partial u_{1}}{\partial x_{2}}=0 \\
\frac{\partial u_{0}}{\partial y_{0}}-\frac{\partial u_{1}}{\partial y_{1}}-\frac{1}{2} \frac{\partial u_{2}}{\partial y_{2}}+\frac{1}{2} \frac{\partial u_{3}}{\partial y_{3}}+\frac{\partial v_{0}}{\partial x_{0}} \\
+\frac{\partial v_{1}}{\partial x_{1}}-\frac{1}{2} \frac{\partial v_{2}}{\partial x_{2}}+\frac{1}{2} \frac{\partial v_{3}}{\partial x_{3}}=0 \\
\frac{\partial u_{3}}{\partial y_{0}}+\frac{\partial u_{2}}{\partial y_{1}}+\frac{1}{2} \frac{\partial u_{0}}{\partial y_{3}}+\frac{1}{2} \frac{1}{\partial y_{2}} \frac{\partial v_{0}}{\partial x_{3}}+\frac{1}{2} \frac{\partial v_{1}}{\partial x_{2}}=0 \\
\frac{\partial u_{1}}{\partial y_{0}}+\frac{\partial u_{0}}{\partial y_{1}}+\frac{1}{2} \frac{\partial u_{2}}{\partial y_{3}}+\frac{1}{2} \frac{\partial u_{3}}{\partial y_{2}}+\frac{\partial v_{1}}{\partial x_{0}} \\
+\frac{\partial v_{0}}{\partial x_{1}}+\frac{1}{2} \frac{\partial v_{2}}{\partial x_{3}}+\frac{1}{2} \frac{\partial v_{3}}{\partial x_{2}}=0 \\
\frac{\partial x_{0}}{\partial y_{2}}+\frac{1}{2} \frac{\partial u_{1}}{\partial y_{3}}+\frac{\partial v_{2}}{\partial x_{0}}
\end{gathered}
$$

The above systems (26) and (27) are corresponding CauchyRiemann systems in $\mathscr{D}(\mathcal{S})$.

On the other hand, the equation $f(z) D_{1}^{*}=0$ of Definition 3 is equivalent to

$$
f_{0} D_{(11)}^{*}=0, \quad f_{0} D_{(12)}^{*}=-f_{1} D_{(11)}^{*} \text {. }
$$


Then,

$$
\begin{aligned}
& g_{0} \frac{\partial}{\partial \overline{z_{0}}}=g_{1} \frac{\partial}{\partial z_{1}}, \quad g_{1} \frac{\partial}{\partial z_{0}}=g_{0} \frac{\partial}{\partial \overline{z_{1}}} \\
& g_{0} \frac{\partial}{\partial \overline{z_{2}}}-g_{1} \frac{\partial}{\partial z_{3}}=-g_{2} \frac{\partial}{\partial \overline{z_{0}}}+g_{3} \frac{\partial}{\partial z_{1}}, \\
& g_{1} \frac{\partial}{\partial z_{2}}-g_{0} \frac{\partial}{\partial \overline{z_{3}}}=-g_{3} \frac{\partial}{\partial z_{0}}+g_{2} \frac{\partial}{\partial \overline{z_{1}}},
\end{aligned}
$$

where

$$
g_{k} \frac{\partial}{\partial \overline{z_{m}}}=\frac{\partial g_{k}}{\partial \overline{z_{m}}}, \quad g_{k} \frac{\partial}{\partial z_{m}}=\frac{\partial g_{k}}{\partial z_{m}} \quad(k, m=0,1,2,3) .
$$

Concretely, the following system is obtained:

$$
\begin{aligned}
& \frac{\partial u_{0}}{\partial x_{0}}-\frac{\partial u_{1}}{\partial x_{1}}=\frac{\partial u_{2}}{\partial x_{2}}+\frac{\partial u_{3}}{\partial x_{3}}, \quad \frac{\partial u_{1}}{\partial x_{0}}+\frac{\partial u_{0}}{\partial x_{1}}=-\frac{\partial u_{2}}{\partial x_{3}}+\frac{\partial u_{3}}{\partial x_{2}}, \\
& \frac{\partial u_{2}}{\partial x_{0}}+\frac{\partial u_{3}}{\partial x_{1}}=\frac{\partial u_{0}}{\partial x_{2}}-\frac{\partial u_{1}}{\partial x_{3}}, \quad \frac{\partial u_{3}}{\partial x_{0}}-\frac{\partial u_{2}}{\partial x_{1}}=\frac{\partial u_{0}}{\partial x_{3}}+\frac{\partial u_{1}}{\partial x_{2}}, \\
& \frac{\partial u_{0}}{\partial y_{0}}-\frac{\partial u_{1}}{\partial y_{1}}-\frac{\partial u_{2}}{\partial y_{2}}-\frac{\partial u_{3}}{\partial y_{3}}=-\frac{\partial v_{0}}{\partial x_{0}}+\frac{\partial v_{1}}{\partial x_{1}}+\frac{\partial v_{2}}{\partial x_{2}}+\frac{\partial v_{3}}{\partial x_{3}} \\
& \frac{\partial u_{1}}{\partial y_{0}}+\frac{\partial u_{0}}{\partial y_{1}}+\frac{\partial u_{2}}{\partial y_{3}}-\frac{\partial u_{3}}{\partial y_{2}}=-\frac{\partial v_{1}}{\partial x_{0}}-\frac{\partial v_{0}}{\partial x_{1}}-\frac{\partial v_{2}}{\partial x_{3}}+\frac{\partial v_{3}}{\partial x_{2}} \\
& \frac{\partial u_{2}}{\partial y_{0}}+\frac{\partial u_{3}}{\partial y_{1}}-\frac{\partial u_{0}}{\partial y_{2}}+\frac{\partial u_{1}}{\partial y_{3}}=-\frac{\partial v_{2}}{\partial x_{0}}-\frac{\partial v_{3}}{\partial x_{1}}+\frac{\partial v_{0}}{\partial x_{2}}-\frac{\partial v_{1}}{\partial x_{3}} \\
& \frac{\partial u_{3}}{\partial y_{0}}-\frac{\partial u_{2}}{\partial y_{1}}-\frac{\partial u_{0}}{\partial y_{3}}-\frac{\partial u_{1}}{\partial y_{2}}=-\frac{\partial v_{3}}{\partial x_{0}}+\frac{\partial v_{2}}{\partial x_{1}}+\frac{\partial v_{0}}{\partial x_{3}}+\frac{\partial v_{1}}{\partial x_{2}} .
\end{aligned}
$$

Similarly, the equation $f(z) D_{2}^{*}=0$ of Definition 3 is equivalent to

$$
f_{0} D_{(21)}^{*}=0, \quad f_{0} D_{(22)}^{*}=-f_{1} D_{(21)}^{*} .
$$

Then,

$$
\begin{aligned}
& g_{0} \frac{\partial}{\partial \overline{z_{0}}}=\frac{1}{2} g_{1} \frac{\partial}{\partial \overline{z_{1}}}, \quad g_{1} \frac{\partial}{\partial z_{0}}=\frac{1}{2} g_{0} \frac{\partial}{\partial z_{1}}, \\
& g_{0} \frac{\partial}{\partial \overline{z_{2}}}-\frac{1}{2} g_{1} \frac{\partial}{\partial \overline{z_{3}}}=-g_{2} \frac{\partial}{\partial \overline{z_{0}}}+\frac{1}{2} g_{3} \frac{\partial}{\partial \overline{z_{1}}} \\
& g_{1} \frac{\partial}{\partial z_{2}}-\frac{1}{2} g_{0} \frac{\partial}{\partial z_{3}}=-g_{3} \frac{\partial}{\partial z_{0}}+\frac{1}{2} g_{2} \frac{\partial}{\partial z_{1}},
\end{aligned}
$$

where

$$
g_{k} \frac{\partial}{\partial \overline{z_{m}}}=\frac{\partial g_{k}}{\partial \overline{z_{m}}}, \quad g_{k} \frac{\partial}{\partial z_{m}}=\frac{\partial g_{k}}{\partial z_{m}} \quad(k, m=0,1,2,3) .
$$

Concretely, the system is obtained as follows:

$$
\begin{gathered}
\frac{\partial u_{0}}{\partial x_{0}}-\frac{\partial u_{1}}{\partial x_{1}}-\frac{1}{2} \frac{\partial u_{2}}{\partial x_{2}}+\frac{1}{2} \frac{\partial u_{3}}{\partial x_{3}}=0 \\
\frac{\partial u_{1}}{\partial x_{0}}+\frac{\partial u_{0}}{\partial x_{1}}-\frac{1}{2} \frac{\partial u_{2}}{\partial x_{3}}-\frac{1}{2} \frac{\partial u_{3}}{\partial x_{2}}=0 \\
\frac{\partial u_{2}}{\partial x_{0}}+\frac{\partial u_{3}}{\partial x_{1}}-\frac{1}{2} \frac{\partial u_{0}}{\partial x_{2}}-\frac{1}{2} \frac{\partial u_{1}}{\partial x_{3}}=0 \\
\frac{\partial u_{3}}{\partial x_{0}}-\frac{\partial u_{2}}{\partial x_{1}}+\frac{1}{2} \frac{\partial u_{0}}{\partial x_{3}}-\frac{1}{2} \frac{\partial u_{1}}{\partial x_{2}}=0 \\
\frac{\partial u_{0}}{\partial y_{0}}-\frac{\partial u_{1}}{\partial y_{1}}-\frac{1}{2} \frac{\partial u_{2}}{\partial y_{2}}+\frac{1}{2} \frac{\partial u_{3}}{\partial y_{3}}+\frac{\partial v_{0}}{\partial x_{0}} \\
-\frac{\partial v_{1}}{\partial x_{1}}-\frac{1}{2} \frac{\partial v_{2}}{\partial x_{2}}+\frac{1}{2} \frac{\partial v_{3}}{\partial x_{3}}=0 \\
\frac{\partial u_{1}}{\partial y_{0}}+\frac{\partial u_{0}}{\partial y_{1}}-\frac{1}{2} \frac{\partial u_{2}}{\partial y_{3}}-\frac{1}{2} \frac{\partial u_{3}}{\partial y_{2}}+\frac{\partial v_{1}}{\partial x_{0}} \\
+\frac{\partial v_{0}}{\partial x_{1}}-\frac{1}{2} \frac{\partial v_{2}}{\partial x_{3}}-\frac{1}{2} \frac{\partial v_{3}}{\partial x_{2}}=0 \\
\frac{\partial u_{2}}{\partial y_{0}}+\frac{\partial u_{3}}{\partial y_{1}}-\frac{1}{2} \frac{\partial u_{0}}{\partial y_{2}}+\frac{1}{2} \frac{\partial u_{1}}{\partial y_{3}}+\frac{\partial v_{2}}{\partial x_{0}} \\
+\frac{\partial v_{3}}{\partial x_{1}}-\frac{1}{2} \frac{\partial v_{0}}{\partial x_{2}}+\frac{1}{2} \frac{\partial v_{1}}{\partial x_{3}}=0 \\
\frac{\partial u_{3}}{\partial y_{0}}-\frac{\partial u_{2}}{\partial y_{1}}-\frac{1}{2} \frac{\partial u_{0}}{\partial y_{3}}-\frac{1}{2} \frac{\partial u_{1}}{\partial y_{2}}+\frac{\partial v_{3}}{\partial x_{0}}=0 \\
\frac{\partial v_{2}}{\partial x_{2}}
\end{gathered}
$$

From the systems (24), (27), (31), and (35), the equations $D_{r}^{*} f(z)=0$ and $f(z) D_{r}^{*}=0(r=1,2)$ are different. Therefore, the equations $D_{r}^{*} f(z)=0$ and $f(z) D_{r}^{*}=0(r=$ $1,2)$ should be distinguished as $L_{r}$-regular functions $(r=$ $1,2)$ and $R_{r}$-regular functions $(r=1,2)$ on $\Omega$, respectively. Now the properties of the $L_{r}$-regular function $(r=1,2)$ with values in $\mathscr{D}(\mathcal{S})$ are considered.

\section{Properties of $L_{r}$-Regular Functions $(r=1,2)$ with Values in $\mathscr{D}(\mathcal{S})$}

We consider properties of a $L_{r}$-regular functions $(r=1,2)$ with values in $\mathscr{D}(\mathcal{S})$.

Theorem 4. Let $\Omega$ be an open set in $\mathbb{C}^{2} \times \mathbb{C}^{2}$ and let $f=$ $f_{0}+\varepsilon f_{1}=\left(g_{0}+g_{1} e_{2}\right)+\varepsilon\left(g_{2}+g_{3} e_{2}\right)$ be an $L_{1}$-regular function defined on $\Omega$. Then

$$
D_{1} f=\left\{2\left(\frac{\partial}{\partial \overline{z_{1}}}+\varepsilon \frac{\partial}{\partial \overline{z_{3}}}\right) e_{2}-\left(\frac{\partial}{\partial x_{1}}+\varepsilon \frac{\partial}{\partial y_{1}}\right) e_{1}\right\} f .
$$


Proof. By the system (23), we have

$$
\begin{aligned}
& D_{1} f=D_{(11)} f_{0}+\varepsilon\left(D_{(12)} f_{0}+D_{(11)} f_{1}\right) \\
& =\left(\frac{\partial g_{0}}{\partial z_{0}}+\frac{\partial \overline{g_{1}}}{\partial \overline{z_{1}}}\right)+\left(\frac{\partial g_{1}}{\partial z_{0}}+\frac{\partial \overline{g_{0}}}{\partial \overline{z_{1}}}\right) e_{2} \\
& +\varepsilon\left(\frac{\partial g_{0}}{\partial z_{2}}+\frac{\partial \overline{g_{1}}}{\partial \overline{z_{3}}}+\frac{\partial g_{2}}{\partial z_{0}}+\frac{\partial \overline{g_{3}}}{\partial \overline{z_{1}}}\right) \\
& +\varepsilon\left(\frac{\partial g_{1}}{\partial z_{2}}+\frac{\partial \overline{g_{0}}}{\partial \overline{z_{3}}}+\frac{\partial g_{3}}{\partial z_{0}}+\frac{\partial \overline{g_{2}}}{\partial \overline{z_{1}}}\right) e_{2} \\
& =\left(\frac{\partial g_{0}}{\partial \overline{z_{0}}}+\frac{\partial u_{1}}{\partial x_{1}}-\frac{\partial u_{0}}{\partial x_{1}} e_{1}+\frac{\partial \overline{g_{1}}}{\partial \overline{z_{1}}}\right) \\
& +\left(\frac{\partial g_{1}}{\partial \overline{z_{0}}}+\frac{\partial u_{3}}{\partial x_{1}}-\frac{\partial u_{2}}{\partial x_{1}} e_{1}+\frac{\partial \overline{g_{0}}}{\partial \overline{z_{1}}}\right) e_{2} \\
& +\varepsilon\left(\frac{\partial g_{0}}{\partial \overline{z_{2}}}+\frac{\partial u_{1}}{\partial y_{1}}-\frac{\partial u_{0}}{\partial y_{1}} e_{1}+\frac{\partial \overline{g_{1}}}{\partial \overline{z_{3}}}\right. \\
& \left.+\frac{\partial g_{2}}{\partial \overline{z_{0}}}+\frac{\partial v_{1}}{\partial x_{1}}-\frac{\partial v_{0}}{\partial x_{1}} e_{1}+\frac{\partial \overline{g_{3}}}{\partial \overline{z_{1}}}\right) \\
& +\varepsilon\left(\frac{\partial g_{1}}{\partial \overline{z_{2}}}+\frac{\partial u_{3}}{\partial y_{1}}-\frac{\partial u_{2}}{\partial y_{1}} e_{1}+\frac{\partial \overline{g_{0}}}{\partial \overline{z_{3}}}\right. \\
& \left.+\frac{\partial g_{3}}{\partial \overline{z_{0}}}+\frac{\partial v_{3}}{\partial x_{1}}-\frac{\partial v_{2}}{\partial x_{1}} e_{1}+\frac{\partial \overline{g_{2}}}{\partial \overline{z_{1}}}\right) e_{2} \\
& =\left(\frac{\partial u_{1}}{\partial x_{1}}-\frac{\partial u_{0}}{\partial x_{1}} e_{1}+2 \frac{\partial \overline{g_{1}}}{\partial \overline{z_{1}}}\right) \\
& +\left(\frac{\partial u_{3}}{\partial x_{1}}-\frac{\partial u_{2}}{\partial x_{1}} e_{1}+2 \frac{\partial \overline{g_{0}}}{\partial \overline{z_{1}}}\right) e_{2} \\
& +\varepsilon\left(\frac{\partial u_{1}}{\partial y_{1}}-\frac{\partial u_{0}}{\partial y_{1}} e_{1}+2 \frac{\partial \overline{g_{1}}}{\partial \overline{z_{3}}}\right. \\
& \left.+\frac{\partial v_{1}}{\partial x_{1}}-\frac{\partial v_{0}}{\partial x_{1}} e_{1}+2 \frac{\partial \overline{g_{3}}}{\partial \overline{z_{1}}}\right) \\
& +\varepsilon\left(\frac{\partial u_{3}}{\partial y_{1}}-\frac{\partial u_{2}}{\partial y_{1}} e_{1}+2 \frac{\partial \overline{g_{0}}}{\partial \overline{z_{3}}}\right. \\
& \left.+\frac{\partial v_{3}}{\partial x_{1}}-\frac{\partial v_{2}}{\partial x_{1}} e_{1}+2 \frac{\partial \overline{g_{2}}}{\partial \overline{z_{1}}}\right) e_{2} \\
& =\left\{2\left(\frac{\partial}{\partial \overline{z_{1}}}+\varepsilon \frac{\partial}{\partial \overline{z_{3}}}\right) e_{2}-\left(\frac{\partial}{\partial x_{1}}+\varepsilon \frac{\partial}{\partial y_{1}}\right) e_{1}\right\} f \text {. }
\end{aligned}
$$

Therefore, we obtain

$$
D_{1} f=\left\{2\left(\frac{\partial}{\partial \overline{z_{1}}}+\varepsilon \frac{\partial}{\partial \overline{z_{3}}}\right) e_{2}-\left(\frac{\partial}{\partial x_{1}}+\varepsilon \frac{\partial}{\partial y_{1}}\right) e_{1}\right\} f
$$

Theorem 5. Let $\Omega$ be an open set in $\mathbb{C}^{2} \times \mathbb{C}^{2}$ and $f=f_{0}+\varepsilon f_{1}=$ $\left(g_{0}+g_{1} e_{2}\right)+\varepsilon\left(g_{2}+g_{3} e_{2}\right)$ be an $L_{2}$-regular function defined on $\Omega$. Then

$$
D_{2} f=\left\{\left(\frac{\partial}{\partial z_{1}}+\varepsilon \frac{\partial}{\partial z_{3}}\right) e_{2}-\left(\frac{\partial}{\partial x_{1}}+\varepsilon \frac{\partial}{\partial y_{1}}\right) e_{1}\right\} f .
$$

Proof. By the system (26), we have

$$
\begin{aligned}
& D_{2} f=D_{(21)} f_{0}+\varepsilon\left(D_{(22)} f_{0}+D_{(21)} f_{1}\right) \\
& =\left(\frac{\partial g_{0}}{\partial z_{0}}+\frac{1}{2} \frac{\partial \overline{g_{1}}}{\partial z_{1}}\right)+\left(\frac{\partial g_{1}}{\partial z_{0}}+\frac{1}{2} \frac{\partial \overline{g_{0}}}{\partial z_{1}}\right) e_{2} \\
& +\varepsilon\left(\frac{\partial g_{0}}{\partial z_{2}}+\frac{1}{2} \frac{\partial \overline{g_{1}}}{\partial z_{3}}+\frac{\partial g_{2}}{\partial z_{0}}+\frac{1}{2} \frac{\partial \overline{g_{3}}}{\partial z_{1}}\right) \\
& +\varepsilon\left(\frac{\partial g_{1}}{\partial z_{2}}+\frac{1}{2} \frac{\partial \overline{g_{0}}}{\partial z_{3}}+\frac{\partial g_{3}}{\partial z_{0}}+\frac{1}{2} \frac{\partial \overline{g_{2}}}{\partial z_{1}}\right) e_{2} \\
& =\left(\frac{\partial g_{0}}{\partial \overline{z_{0}}}+\frac{\partial u_{1}}{\partial x_{1}}-\frac{\partial u_{0}}{\partial x_{1}} e_{1}+\frac{1}{2} \frac{\partial \overline{g_{1}}}{\partial z_{1}}\right) \\
& +\left(\frac{\partial g_{1}}{\partial \overline{z_{0}}}+\frac{\partial u_{3}}{\partial x_{1}}-\frac{\partial u_{2}}{\partial x_{1}} e_{1}+\frac{1}{2} \frac{\partial \overline{g_{0}}}{\partial z_{1}}\right) e_{2} \\
& +\varepsilon\left(\frac{\partial g_{0}}{\partial \overline{z_{2}}}+\frac{\partial u_{1}}{\partial y_{1}}-\frac{\partial u_{0}}{\partial y_{1}} e_{1}+\frac{1}{2} \frac{\partial \overline{g_{1}}}{\partial z_{3}}\right. \\
& \left.+\frac{\partial g_{2}}{\partial \overline{z_{0}}}+\frac{\partial v_{1}}{\partial x_{1}}-\frac{\partial v_{0}}{\partial x_{1}} e_{1}+\frac{1}{2} \frac{\partial \overline{g_{3}}}{\partial z_{1}}\right) \\
& +\varepsilon\left(\frac{\partial g_{1}}{\partial \overline{z_{2}}}+\frac{\partial u_{3}}{\partial y_{1}}-\frac{\partial u_{2}}{\partial y_{1}} e_{1}+\frac{1}{2} \frac{\partial \overline{g_{0}}}{\partial z_{3}}\right. \\
& \left.+\frac{\partial g_{3}}{\partial \overline{z_{0}}}+\frac{\partial v_{3}}{\partial x_{1}}-\frac{\partial v_{2}}{\partial x_{1}} e_{1}+\frac{1}{2} \frac{\partial \overline{g_{2}}}{\partial z_{1}}\right) e_{2} \\
& =\left(\frac{\partial u_{1}}{\partial x_{1}}-\frac{\partial u_{0}}{\partial x_{1}} e_{1}+\frac{\partial \overline{g_{1}}}{\partial z_{1}}\right) \\
& +\left(\frac{\partial u_{3}}{\partial x_{1}}-\frac{\partial u_{2}}{\partial x_{1}} e_{1}+\frac{\partial \overline{g_{0}}}{\partial z_{1}}\right) e_{2} \\
& +\varepsilon\left(\frac{\partial u_{1}}{\partial y_{1}}-\frac{\partial u_{0}}{\partial y_{1}} e_{1}+\frac{\partial \overline{g_{1}}}{\partial z_{3}}\right. \\
& \left.+\frac{\partial v_{1}}{\partial x_{1}}-\frac{\partial v_{0}}{\partial x_{1}} e_{1}+\frac{\partial \overline{g_{3}}}{\partial z_{1}}\right) \\
& +\varepsilon\left(\frac{\partial u_{3}}{\partial y_{1}}-\frac{\partial u_{2}}{\partial y_{1}} e_{1}+\frac{\partial \overline{g_{0}}}{\partial z_{3}}\right. \\
& \left.+\frac{\partial v_{3}}{\partial x_{1}}-\frac{\partial v_{2}}{\partial x_{1}} e_{1}+\frac{\partial \overline{g_{2}}}{\partial z_{1}}\right) e_{2} \\
& =\left\{\left(\frac{\partial}{\partial z_{1}}+\varepsilon \frac{\partial}{\partial z_{3}}\right) e_{2}-\left(\frac{\partial}{\partial x_{1}}+\varepsilon \frac{\partial}{\partial y_{1}}\right) e_{1}\right\} f .
\end{aligned}
$$

Therefore, we obtain the following equation:

$$
D_{2} f=\left\{\left(\frac{\partial}{\partial z_{1}}+\varepsilon \frac{\partial}{\partial z_{3}}\right) e_{2}-\left(\frac{\partial}{\partial x_{1}}+\varepsilon \frac{\partial}{\partial y_{1}}\right) e_{1}\right\} f .
$$


Proposition 6. From properties of differential operators, the following equations are obtained:

$$
\begin{aligned}
& D_{(1 r)} p_{r-1}=2, D_{(2 r)} p_{r-1}=1, \\
& D_{(1 r)}^{*} p_{r-1}=-1, \quad D_{(2 r)}^{*} p_{r-1}=0, \\
& D_{(1 r)}^{*} p_{r-1}^{*}=2, \quad D_{(2 r)}^{*} p_{r-1}^{*}=1, \\
& D_{(r 1)} p_{1}=D_{(r 1)}^{*} p_{1}=D_{(r 1)} p_{1}^{*}=D_{(r 1)}^{*} p_{1}^{*} \\
&=D_{(r 2)} p_{0}=D_{(r 2)}^{*} p_{0}=D_{(r 2)} p_{0}^{*} \\
&=D_{(r 2)}^{*} p_{0}^{*}=0 \quad(r=1,2) .
\end{aligned}
$$

Proof. By properties of the power of dual split quaternions and derivatives on $\mathscr{D}(\mathcal{S})$, the following derivatives are obtained:

$$
\begin{aligned}
D_{(11)} p_{0}= & \frac{1}{2}\left(\frac{\partial}{\partial x_{0}}-\frac{\partial}{\partial x_{1}} e_{1}+\frac{\partial}{\partial x_{2}} e_{2}+\frac{\partial}{\partial x_{3}} e_{3}\right) \\
& \times\left(x_{0}+x_{1} e_{1}+x_{2} e_{2}+x_{3} e_{3}\right)=2 \\
D_{(22)}^{*} p_{1}= & \frac{1}{2}\left(\frac{\partial}{\partial y_{0}}+\frac{\partial}{\partial y_{1}} e_{1}-\frac{1}{2} \frac{\partial}{\partial y_{2}} e_{2}+\frac{1}{2} \frac{\partial}{\partial y_{3}} e_{3}\right) \\
& \times\left(y_{0}+y_{1} e_{1}+y_{2} e_{2}+y_{3} e_{3}\right)=0 \\
D_{(11)} p_{0}^{*}= & \frac{1}{2}\left(\frac{\partial}{\partial x_{0}}-\frac{\partial}{\partial x_{1}} e_{1}+\frac{\partial}{\partial x_{2}} e_{2}+\frac{\partial}{\partial x_{3}} e_{3}\right) \\
& \times\left(x_{0}-x_{1} e_{1}-x_{2} e_{2}-x_{3} e_{3}\right)=-1 .
\end{aligned}
$$

The other equations are calculated using a similar method, and the above equations are obtained.

Theorem 7. Let $\Omega$ be an open set in $\mathbb{C}^{2} \times \mathbb{C}^{2}$ and let $f(z)$ be a function on $\Omega$ with values in $\mathscr{D}(\mathcal{S})$. Then the power $z^{n}$ of $z$ in $\mathscr{D}(\mathcal{S})$ is not an $L_{1}$-regular function but an $L_{2}$-regular function on $\Omega$, where $n \in \mathbb{N}$.

Proof. From the definition of the $L_{r}$-regular function $(r=$ $1,2)$ on $\Omega$ and Proposition 6, we may consider whether the power $z^{n}$ of $z$ in $\mathscr{D}(\mathcal{S})$ satisfies the equation $D_{r}^{*} z^{n}=0(r=$ $1,2)$. Since $D_{(11)}^{*} p_{0}=2$,

$$
\begin{aligned}
D_{1}^{*} z^{n} & =\left(D_{(11)}^{*}+\varepsilon D_{(12)}^{*}\right)\left(p_{0}^{n}+\varepsilon \sum_{k=1}^{n} p_{0}^{n-k} p_{1} p_{0}^{k-1}\right) \\
& =D_{(11)}^{*} p_{0}^{n}+\varepsilon\left(\sum_{k=1}^{n} D_{(11)}^{*} p_{0}^{n-k} p_{1} p_{0}^{k-1}+D_{(12)}^{*} p_{0}^{n}\right) \neq 0 .
\end{aligned}
$$

Hence, the power $z^{n}$ of $z$ is not $L_{1}$-regular on $\Omega$. On the other hand, from the equations in Proposition 6, we have $D_{(21)}^{*} p_{0}=$ $0, D_{(21)}^{*} p_{1}=0$, and $D_{(22)}^{*} p_{0}=0$. Then,

$$
D_{2}^{*} z^{n}=D_{(21)}^{*} p_{0}^{n}+\varepsilon\left(\sum_{k=1}^{n} D_{(21)}^{*} p_{0}^{n-k} p_{1} p_{0}^{k-1}+D_{(22)}^{*} p_{0}^{n}\right)=0
$$

Therefore, by the definition of the $L_{r}$-regular function $(r=$ $1,2)$ on $\Omega$, a power $z^{n}$ of $z$ is $L_{2}$-regular on $\Omega$.

\section{Conflict of Interests}

The authors declare that there is no conflict of interests regarding the publication of this paper.

\section{Acknowledgments}

The second author was supported by the Basic Science Research Program through the National Research Foundation of Korea (NRF) funded by the Ministry of Science, ICT, and Future Planning (2013R1A1A2008978).

\section{References}

[1] I. L. Kantor and A. S. Solodovnikov, Hypercomplex Numbers: An Elementary Introduction to Algebras, Springer, New York, NY, USA, 1989.

[2] J. Cockle, "On systems of algebra involving more than one imaginary," Philosophical Magazine III, vol. 35, no. 238, pp. 434435, 1849.

[3] M. Özdemir and A. A. Ergin, "Rotations with unit timelike quaternions in Minkowski 3-space," Journal of Geometry and Physics, vol. 56, no. 2, pp. 322-336, 2006.

[4] L. Kula and Y. Yayli, "Split quaternions and rotations in semi Euclidean space $\mathbb{E}_{2}^{4}$," Journal of the Korean Mathematical Society, vol. 44, no. 6, pp. 1313-1327, 2007.

[5] D. C. Brody and E.-M. Graefe, "On complexified mechanics and coquaternions," Journal of Physics A: Mathematical and Theoretical, vol. 44, no. 7, article 072001, 2011.

[6] I. Frenkel and M. Libine, "Split quaternionic analysis and separation of the series for $\operatorname{SL}(2, \mathbb{R})$ and $\operatorname{SL}(2, \mathbb{C}) / \operatorname{SL}(2, \mathbb{R})$," Advances in Mathematics, vol. 228, no. 2, pp. 678-763, 2011.

[7] J.-i. Inoguchi, “Timelike surfaces of constant mean curvature in Minkowski 3-space," Tokyo Journal of Mathematics, vol. 21, no. 1, pp. 141-152, 1998.

[8] B. Kenwright, "A beginners guide to dual-quaternions: what they are, how they work, and how to use them for 3D character hierarchies," in Proceedings of the 20th International Conferences on Computer Graphics, Visualization and Computer Vision, pp. $1-10,2012$.

[9] E. Pennestrì and R. Stefanelli, "Linear algebra and numerical algorithms using dual numbers," Multibody System Dynamics, vol. 18, no. 3, pp. 323-344, 2007.

[10] L. H. Son, "An extension problem for solutions of partial differential equations in $\mathbb{R}^{n}$," Complex Variables: Theory and Application, vol. 15, no. 2, pp. 87-92, 1990.

[11] L. H. Son, "Extension problem for functions with values in a Clifford algebra," Archiv der Mathematik, vol. 55, no. 2, pp. 146$150,1990$.

[12] J. Kajiwara, X. D. Li, and K. H. Shon, "Regeneration in complex, quaternion and Clifford analysis," in International Colloquium on Finite or Infinite DimensionalComplex Analysis and Its Applications, vol. 2 of Advances in Complex Analysis and Its Applications no. 9, pp. 287-298, Kluwer Academic Publishers, Hanoi, Vietnam, 2004.

[13] J. Kajiwara, X. D. Li, and K. H. Shon, "Function spaces in complex and Clifford analysis," in International Colloquium 
on Finite Or Infinite Dimensional Complex Analysis and Its Applications, vol. 14 of Inhomogeneous Cauchy Riemann System of Quaternion and Clifford Analysis in Ellipsoid, pp. 127-155, Hue University, Hue, Vietnam, 2006.

[14] J. E. Kim, S. J. Lim, and K. H. Shon, "Regular functions with values in ternary number system on the complex Clifford analysis," Abstract and Applied Analysis, vol. 2013, Article ID 136120, 7 pages, 2013.

[15] J. E. Kim, S. J. Lim, and K. H. Shon, "Regularity of functions on the reduced quaternion field in Clifford analysis," Abstract and Applied Analysis, vol. 2014, Article ID 654798, 8 pages, 2014.

[16] S. J. Lim and K. H. Shon, "Hyperholomorphic fucntions and hyperconjugate harmonic functions of octonion variables," Journal of Inequalities and Applications, vol. 2013, article 77, 2013.

[17] S. J. Lim and K. H. Shon, "Dual quaternion functions and its applications," Journal of Applied Mathematics, vol. 2013, Article ID 583813, 6 pages, 2013.

[18] J. E. Kim, S. J. Lim, and K. H. Shon, "Taylor series of functions with values in dual quaternion," Journal of the Korean Society of Mathematical Education B-The Pure and Applied Mathematics, vol. 20, no. 4, pp. 251-258, 2013. 


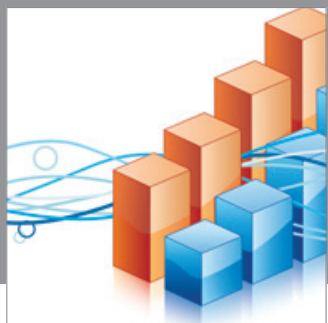

Advances in

Operations Research

mansans

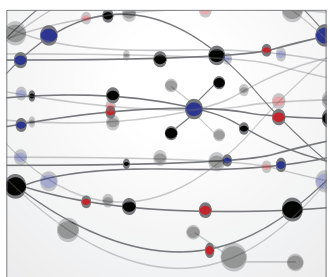

The Scientific World Journal
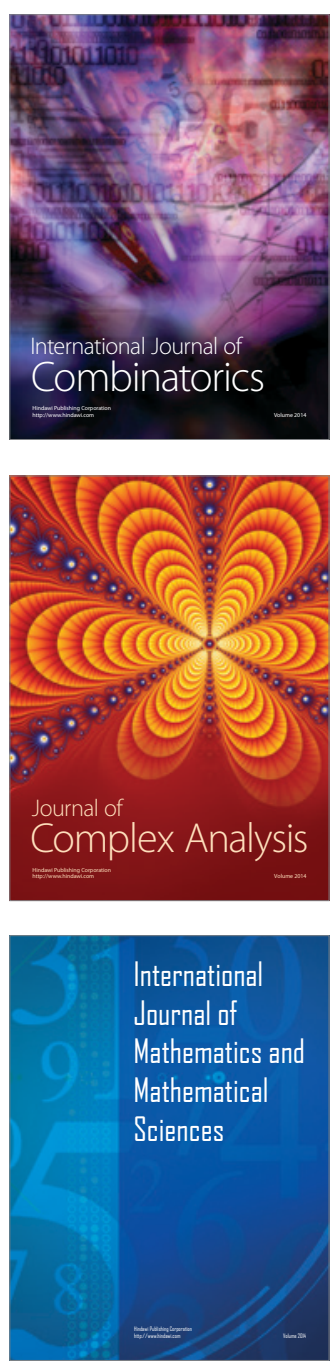
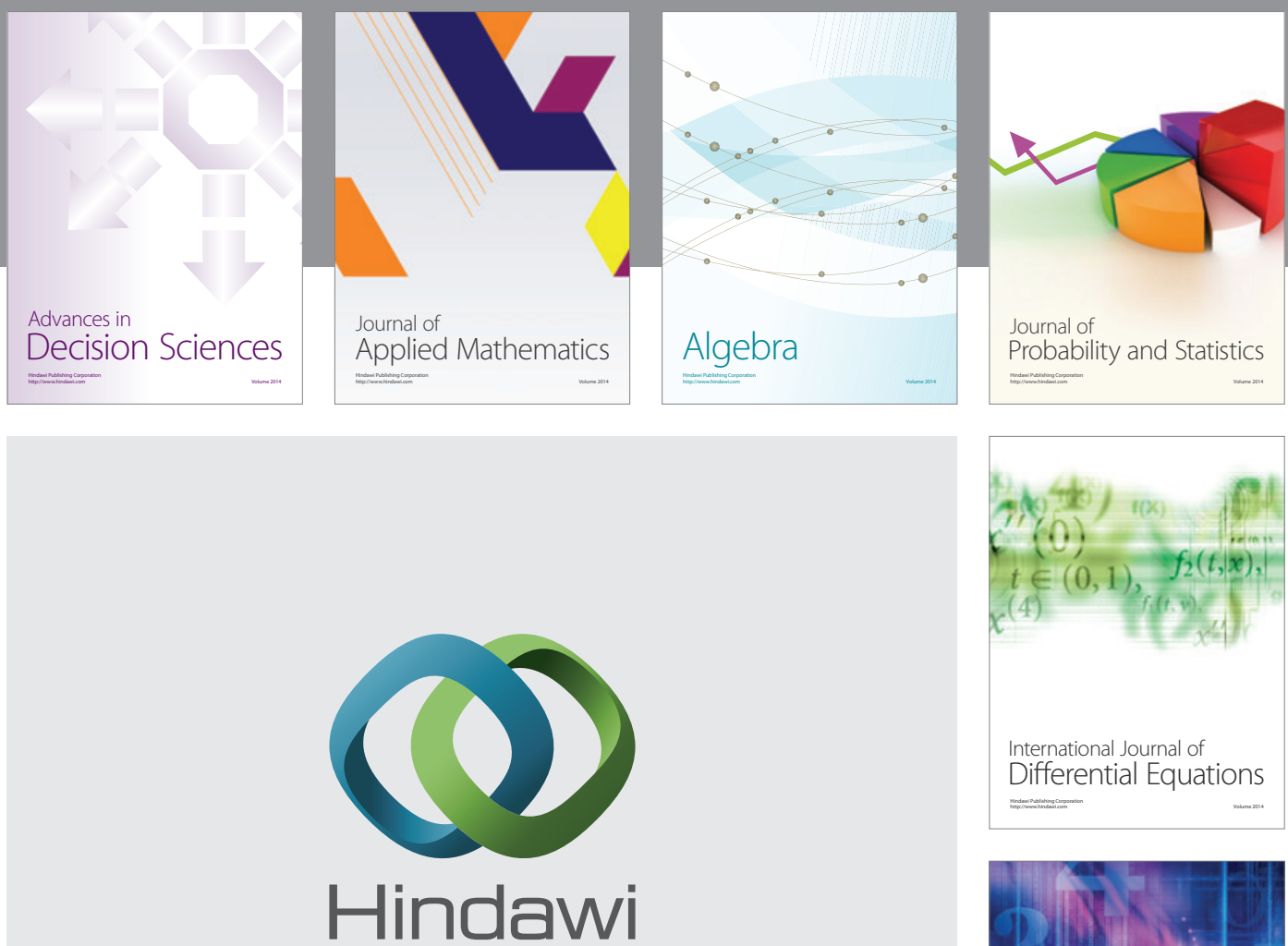

Submit your manuscripts at http://www.hindawi.com
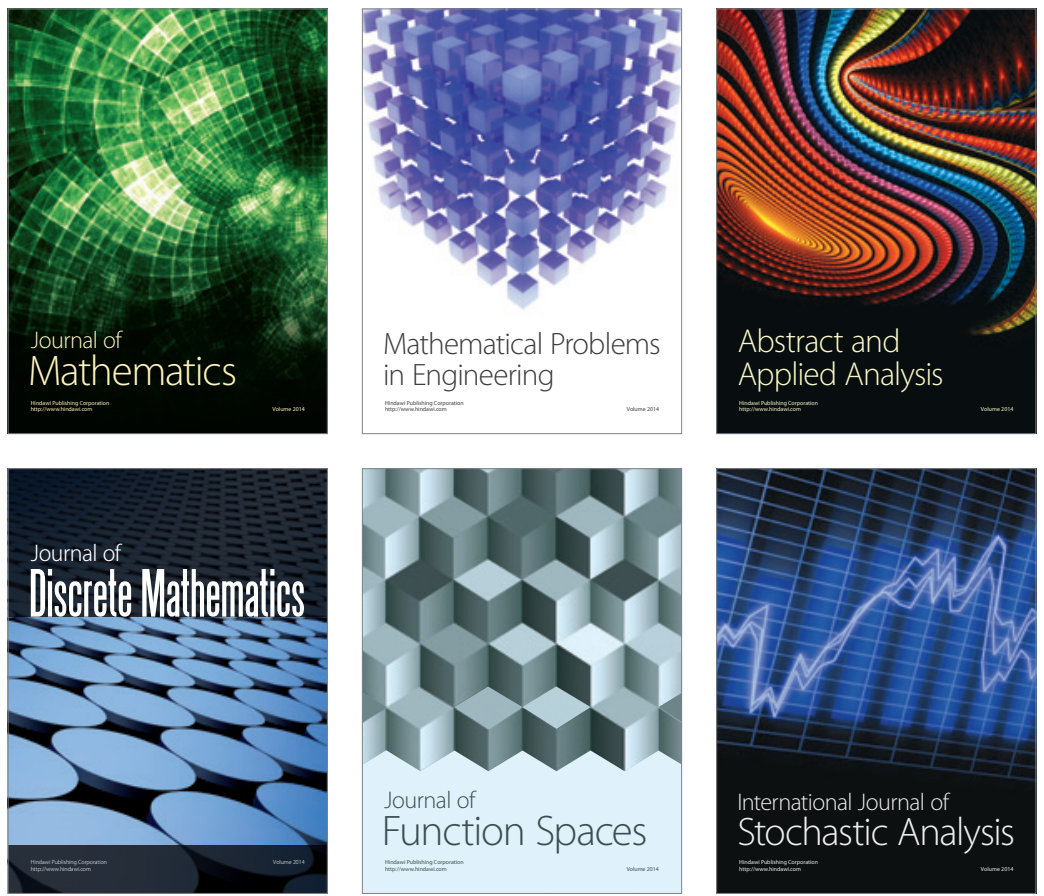

Journal of

Function Spaces

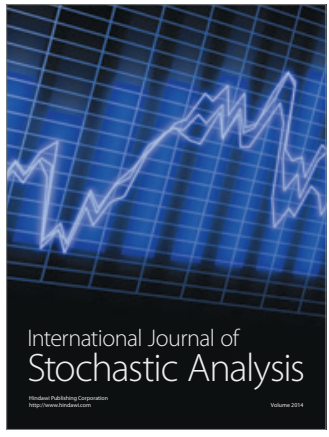

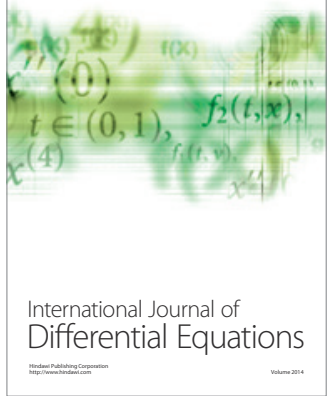
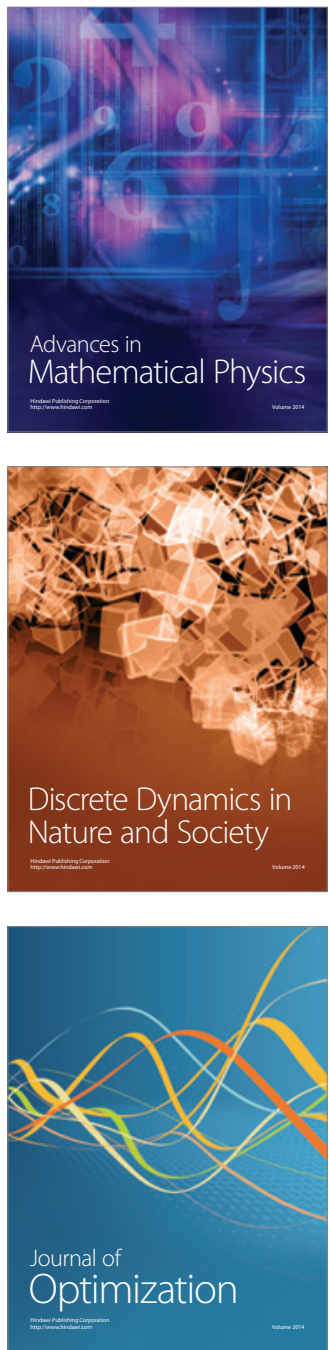